\title{
Plantas asociadas a los bosques de Abies guatemalensis (Pinaceae) del occidente de Guatemala
}

\author{
José Vicente Martínez Arévalo \\ Subárea de Ciencias Biológicas, Facultad de Agronomía, Universidad de San Carlos de Guatemala. Edificio T-8, \\ Ciudad Universitaria, zona 12, Guatemala; josevm2000@yahoo.com
}

\author{
Recibido 14-II-2012. Corregido 30-VII-2012. Aceptado 28-VIII-2012.
}

\begin{abstract}
Plants associated to Abies guatemalensis (Pinaceae) forests in Western Guatemala. The fragments of Abies guatemalensis forests in Western Guatemala are the reservoirs of plant species that have been poorly documented, missing the opportunity to expand the knowledge of the local flora and its use in conservation planning. To assess this, a floristic study was done in areas between $2950-3360$ masl in Western Guatemala between 2010-2011. Ten locations were sampled: in each a $500 \mathrm{~m}^{2}$ plot was surveyed, and plants were classified in four strata by plant height $(0.05-30 \mathrm{~m})$. A total of 119 species, 92 genera and 50 families in four divisions were found. The families with more species were Asteraceae, Poaceae, Rosaceae, Lamiaceae, Apiaceae and Solanaceae, and the most abundant genera were Salvia, Alchemilla and Bidens. The number of species found by strata was: 33 (low herbaceous), 49 (high herbaceous), 30 (shrubs) and seven in the tree strata. Regarding geographical distribution, the biggest species group detected was from central Mexico to Central America with 67\%, which compared to the forests of A. guatemalensis in central and Southern Mexico, showed high floristic affinity, especially at the family and genus level. However, even having families and genera in common in the general structure of the fir forests, their floristic particularities should be taken into account when making management and conservation plans, because these are influenced by soil, latitude and microclimate conditions. Rev. Biol. Trop. 61 (1): 321-333. Epub 2013 March 01.
\end{abstract}

Key words: flora, conservation, floristic richness, fir, Abies guatemalensis, geographical distribution.

Los estudios florísticos y de vegetación son importantes porque permiten aumentar el conocimiento de las especies y biodiversidad de una región o país (Knapp et al. 2001, Sánchez \& Grados 2007), en especial aquellas con valor ecológico y económico (Zamora 2003), también sirve para crear bases de datos (Heywood 2004) y para abordar estudios posteriores de recursos vegetales (Olguín 2008, López-Sandoval et al. 2010). Aunque hace falta ser reconocido por algunos biólogos de la conservación (Prima \& Alfonso 2002), el conocimiento de la flora es fundamental para la justificación y desarrollo de directrices de conservación (Knapp et al. 2001, Ejtehadi et al. 2005), especialmente en áreas fragmentadas (Ponce et al. 2006, Simonetti 2006, Bouroncle
2008, Stevenson \& Rodríguez 2008) como el caso de los bosques de Abies guatemalensis Rehder (abeto) (Melgar 2003, Strandby et al . 2009). El conocimiento de las especies vegetales de los bosques templados de Guatemala está escasamente documentado (Bermúdez \& Sánchez 2000), y como lo ha señalado Islebe et al. (1994), hay carencia de información detallada sobre la composición y estructura de las comunidades montanas guatemaltecas, lo que limita las investigaciones comparativas del tipo ecológico con áreas adyacentes, por ejemplo con el sur y centro de México. Es importante un mayor conocimiento florístico sistematizado de la región, para ayudar en la conservación (Toledo et al. 2005) ya que en esta, hay varias especies incluidas en la lista roja de CITES, 
ente ellas A. guatemalensis (Melgar 2003, CONAP 2009). Deben reconocerse avances en los últimos años en la documentación de las especies vegetales de los bosques templados y fríos de Guatemala. Veliz (1998), Viñals et al. (2001), Veliz et al. (2001) han contribuido a la publicación de catálogos de la flora montana y cumbres de los volcanes del occidente y el centro de Guatemala, que permite tener referencia del tipo de vegetación existente en el área de interés de este estudio. También Islebe et al. (1994) estudiaron las especies leñosas de la sierra de los Cuchumatanes y de la cadena montañosa de Guatemala. Para los bosques de A. guatemalensis una de las pocas referencias documentadas, es el trabajo de González \& Castañeda (1983) en las comunidades vegetales de abeto del occidente del Guatemala.

Se tiene conocimiento que la flora de los bosques montanos del occidente de Guatemala, comparten afinidad florística con los del centro y sur de México, debido a la historia biogeográfica similar (Shuster \& Bonis 2008, Luna-Vega 2008) y condiciones ecológicas parecidas, tal es el caso de los bosques de $A$. religiosa (Kunth) Schltdl. y A. guatemalensis (Jaramillo-Correa et al. 2008, Strandby et al. 2009, Rasmussen et al. 2010). Velázquez \& Islebe (1995) demostraron la afinidad florística entre las montañas del centro de México y Guatemala, al encontrar que pertenecen a Megaméxico 2, área fitogeográfica propuesta por Rzedowski (1991), que comprende Mesoamérica. Aunque estos tipos de vegetación han sido influenciados de manera similar por factores histórico-climáticos, responden diferencialmente a factores ecológicos, lo que se refleja en las características florísticas que los tipifican como unidades independientes (Velázquez \& Islebe 1995). Entre los trabajos florísticos y de vegetación de Abies para el centro y sur de México, están los de Sánchez et al. (1991), Ávila \& López (2001), SánchezGonzález et al. (2005), Sánchez et al. (2006) que pueden ser de utilidad para la comparación con la flora de los bosques similares de Guatemala. Por esto, el objetivo de este artículo es contribuir al conocimiento de las especies y la distribución geográfica de las plantas de los bosques de $A$. guatemalensis de la parte alta de las montañas de la cadena volcánica occidental de Guatemala.

\section{MATERIALES Y MÉTODOS}

Área de estudio: Se ubicó en el departamento de San Marcos entre $2800-3$ 400m de altitud, en el occidente de Guatemala (14'55'32"

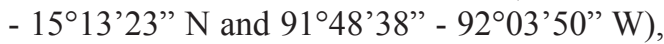
que colinda con Chiapas, México. Con base en Holdridge (1978) el área de estudio comprendió las zonas de vida Bosque muy húmedo montano bajo Subtropical (Bmh-MB) y Bosque húmedo montano bajo Subtropical (Bh-MB), cuya vegetación arbórea está representada por Alnus jorullensis, Arbutus xalapensis, Chiranthodendron pentadactilylon, Neocupressus lusitanica, Ostrya spp., Pinus ayacahuite, $P$. hartwegii, P. pseudostrobus, Prunus serotina, Quercus spp. y Buddleja spp. De acuerdo con Morrone (2001) está ubicada en la Región Biogeográfica Neotropical, Provincia de Chiapas. El clima es de templado a frío con temperatura media anual de $15^{\circ} \mathrm{C}$, que llega a descender por la noche por debajo de $\operatorname{los} 0^{\circ} \mathrm{C}$, presentándose heladas, sobre todo de noviembre a marzo. La humedad relativa promedio es del $75 \%$, una precipitación promedio de $1300 \mathrm{~mm}$ anuales (INSIVUMEH 2003). Fisiográficamente son montañas cubiertas de rocas y cenizas volcánicas recientes, provenientes de los volcanes que rodean el área, como el Tacana con 4093m de altitud y poca actividad volcánica y el Tajumulco con 4220m de altitud sin actividad volcánica actual. El orden de suelos dominante es el Andosol (FAO 2007). Son suelos de 75-99cm de profundidad, ácidos, de textura franca, color negro a gris, con moderada porosidad y con alta erosionabilidad (Simmons et al. 1959). El área es quebrada con pendientes pronunciadas en su mayoría, las tierras son utilizadas para pastoreo de ovejas, cultivos y áreas de bosque. En el cuadro 1 se muestra las localidades y la ubicación donde se efectuaron los levantamientos florísticos. 
CUADRO 1

Bosques de abeto (Abies guatemalensis), en donde se realizó el levantamiento florístico

TABLE 1

Fir forests (Abies guatemalensis), where the floristic uprising was conducted

\begin{tabular}{|c|c|c|c|c|c|c|}
\hline \multirow{2}{*}{ No. } & \multirow{2}{*}{ Localidad } & \multirow{2}{*}{ Municipio } & \multicolumn{2}{|c|}{ Coordenadas } & \multirow{2}{*}{ Altitud msnm } & \multirow{2}{*}{ Área ha } \\
\hline & & & Latitud N & Longitud W & & \\
\hline 1 & Camba & Sibinal & $15^{\circ} 10^{\prime} 17^{\prime \prime}$ & $92^{\circ} 03^{\prime} 04^{\prime \prime}$ & 3300 & 11.80 \\
\hline 2 & Los Cuervos & Ixchiguan & $15^{\circ} 10^{\prime} 13^{\prime \prime}$ & $91^{\circ} 56^{\prime} 52^{\prime \prime}$ & 3360 & 3.07 \\
\hline 3 & Bosque Ixcamal & San Marcos & $15^{\circ} 00^{\prime} 35^{\prime \prime}$ & $91^{\circ} 48^{\prime} 48^{\prime \prime}$ & 2950 & 37.10 \\
\hline 4 & Bosque El Grande & San José Ojetenam & $15^{\circ} 13^{\prime} 23^{\prime \prime}$ & $91^{\circ} 58^{\prime} 01^{\prime \prime}$ & 3134 & 8.00 \\
\hline 5 & Las Nubes & San José Ojetenam & $15^{\circ} 12^{\prime} 55^{\prime \prime}$ & $91^{\circ} 57^{\prime} 24^{\prime \prime}$ & 3370 & 10.00 \\
\hline 6 & Las Ventanas & San José Ojetenam & $15^{\circ} 12^{\prime} 34^{\prime \prime}$ & $91^{\circ} 57^{\prime} 54^{\prime \prime}$ & 3206 & 40.00 \\
\hline 7 & Canatzaj & Tacana & $15^{\circ} 12^{\prime} 07^{\prime \prime}$ & $91^{\circ} 57^{\prime} 13^{\prime \prime}$ & 3200 & 5.00 \\
\hline 8 & Flor de Mayo & Tacana & $15^{\circ} 10^{\prime} 49^{\prime \prime}$ & $91^{\circ} 57^{\prime} 39^{\prime \prime}$ & 3245 & 4.00 \\
\hline 9 & Toribio & Tacana & $15^{\circ} 10^{\prime} 40^{\prime \prime}$ & $91^{\circ} 58^{\prime} 06^{\prime \prime}$ & 3150 & 2.00 \\
\hline 10 & San Luis & Tacana & $15^{\circ} 10^{\prime} 44^{\prime \prime}$ & $91^{\circ} 57^{\prime} 56^{\prime \prime}$ & 3200 & 4.00 \\
\hline
\end{tabular}

Metodología de campo: Se realizaron levantamientos florísticos en los bosques de A. guatemalensis y sus áreas de borde, en diez localidades distribuidas en cinco municipios según se indica en el cuadro 1 y en tres épocas (julio-agosto y octubre-noviembre 2010 y febrero-marzo 2011), esto para obtener la mayor parte de las especies a través del año y recoger especímenes con flor y fruto, y su respectiva determinación botánica. Se registró la presencia de las especies, que se utilizó para hacer la lista florística; la altura de las plantas, se clasificaron en cuatro estratos: a) herbáceo inferior $(0.05-0.20 \mathrm{~m}), \mathrm{b})$ herbáceo superior $(0.30-1.00 \mathrm{~m}, \mathrm{c})$ arbustos $(0.50-1.50 \mathrm{~m}$, y d) árboles (2.00-30.00m). En cada localidad se ubicaron cinco parcelas en un gradiente sucesional de etapas de hierbas hasta árboles. La parcela fue de $500 \mathrm{~m}^{2}$, donde se registró la información para árboles, dentro de ella se establecieron cinco sub-parcelas (una en cada esquina y otra en el centro) de $1 \mathrm{~m}^{2}$ para hierbas y una sub-parcela en la esquina superior derecha de la parcela grande de $16 \mathrm{~m}^{2}$ para arbustos. Se recolectaron muestras de herbario, las que se documentaron con información morfológica de las plantas y descripción del lugar, se colocaron en bolsas con alcohol para su preservación, y de esta forma se enviaron al herbario.
Metodología de herbario: La determinación botánica se realizó en el herbario BIGU de la Escuela Biológica de la Facultad de Ciencias Químicas y Farmacia de la Universidad de San Carlos de Guatemala, donde se secó, preparó y herborizó los especímenes de cada especie. La determinación se hizo con base en Mosses of Guatemala (Bertram 1949) Flora of Guatemala (Standley \& Steyermark 1947-1977), y Ferns and fern allies of Guatemala (Stolze 1981) con la actualización de familias y nombres científicos de la Flora de Nicaragua (Stevens 2001). Con esto se elaboró una lista ordenada por división, clase, familia género y especie, como se muestra en el apéndice 1, y se elaboró un cuadro resumen. Muestras herborizadas de las especies quedaron depositadas en el herbario BIGU.

De acuerdo con la información geográfica existente en la base de datos de Trópicos (www. tropicos.org), se hizo la siguiente clasificación de distribución de los taxones: 1: Centro de México hasta Centroamérica; 2: México hasta Sudamérica; 3: Estados Unidos de Norteamérica hasta Centroamérica; 4: América; 5: Amplia distribución y 6: Guatemala hasta Sudamérica. La información se presenta por medio de una figura. 


\section{RESULTADOS}

Composición florística: Se registraron 119 especies, 92 géneros, 50 familias y cuatro divisiones. La información detallada se presenta en el apéndice $1 \mathrm{y}$ en el cuadro 2 , se hace un resumen de esos resultados. En la columna izquierda, se listan las 19 familias botánicas con dos o más especies que representan el $74 \%$ de la flora registrada, de ellas Asteraceae, Poaceae, Rosaceae, Lamiaceae, Apiaceae y Solanaceae, fueron las más numerosas y hubo 31 familias $(26 \%)$ que presentaron una sola especie. En la segunda columna se muestran los 17 géneros que tuvieron dos o más especies, que representa el 37\% de la flora, de ellos Salvia, Alchemilla y Bidens tuvieron mayor número de especies. En la tercera columna, en la parte superior se indica las especies clasificadas por divisiones:
Magnoliophyta representa el $86 \%$, Polydiophyta, Briophyta y Pinophyta seis, cinco y tres por ciento respectivamente.

En la tercera columna en la parte inferior se clasifican las especies con base en el estrato vertical que ocuparon. El herbáceo superior tuvo el $41 \%$, seguido del herbáceo inferior con $28 \%$, arbustos $25 \%$ y árboles seis por ciento. El estrato herbáceo inferior estuvo compuesto principalmente por musgos [Cyclodictyon albicans (Hedw.) Kuntze, Hypnum amabile (Mitt.) Hampe, Hypopterygium tamariscinum (Hedw.) Brid., Mitthenothamnium reptans (Hedw.) Card., Sematophyllum galipense (C. Mull.) Mitt., y Vesicularia vesicularis (Schwägr.) Broth. var. portoricensis (Brid.) Buck.], plantas con tallos postrados (Erigeron karvinskianus DC., Geranium alpicola Loes., G. repens H.E. Moore, Hydrocotile mexicana

CUADRO 2

Familias, géneros y divisiones de la flora y estratos de vegetación de bosques de abeto (Abies guatemalensis). San Marcos, Guatemala

TABLE 2

Families, genera and divisions of the flora and vegetation strata fir forest (Abies guatemalensis). San Marcos, Guatemala

\begin{tabular}{|c|c|c|c|c|c|}
\hline Familia & Especies & Género & Especies & División & Especies \\
\hline Asteraceae & 24 & Salvia & 5 & Briophyta & 6 \\
\hline Poaceae & 9 & Alchemilla & 4 & Polypodiophyta & 7 \\
\hline Rosaceae & 8 & Bidens & 4 & Pinophyta & 4 \\
\hline Lamiaceae & 7 & Senecio & 3 & Magnoliophyta & 102 \\
\hline Apiaceae & 5 & Solanum & 3 & Total & 119 \\
\hline Solanaceae & 5 & Stevia & 3 & & \\
\hline Hypnaceae & 3 & Arracacia & 2 & & \\
\hline Onagraceae & 3 & Bromus & 2 & & \\
\hline Orobanchaceae & 3 & Buddleja & 2 & & \\
\hline Pinaceae & 3 & Eryngium & 2 & & \\
\hline Araliaceae & 2 & Fuchsia & 2 & & \\
\hline Asparagaceae & 2 & Geranium & 2 & Estrato & Especies \\
\hline Dryopteridaceae & 2 & Lamourouxia & 2 & Herbáceo inferior & 33 \\
\hline Ericaceae & 2 & Pinus & 2 & Altura $0.05-0.20 \mathrm{~m}$ & \\
\hline Fabaceae & 2 & Piptochaetium & 2 & Herbáceo superior & 49 \\
\hline Geraniaceae & 2 & Polystichum & 2 & Altura $0.30-1.00 \mathrm{~m}$ & \\
\hline Rubiaceae & 2 & Verbesina & 2 & Arbustos & 30 \\
\hline Pteridaceae & 2 & 75 géneros & $1 \mathrm{c} / \mathrm{u}$ & Altura $0.50-1.50 \mathrm{~m}$ & \\
\hline Scrophulariaceae & 2 & 92 géneros & 119 & Árboles & 7 \\
\hline 31 familias & $1 \mathrm{c} / \mathrm{u}$ & & & Altura 2.0 a $30.0 \mathrm{~m}$ & \\
\hline Total 50 familias & 119 & & & Total & 119 \\
\hline
\end{tabular}


Schltdl. \& Cham., Smilacina scilloidea M. Martens \& Galeotti, entre las principales), rizomas/tubérculos (Adianthum andicola Liebm., Alchemilla guatemalensis Rothm., A. pectinata Kunth, A. procumbens Rose, A. vulcanica Schltdl. \& Cham., Asplenium costaneum Schltdl. \& Cham., Brachypodium mexicanum (Roem. \& Schult.) Link, Bromus exaltatus Bernh., Campyloneurum xalapense Fée, Cheilanthes sp. Sw., Oxalis alpina (Rose) Rose ex R. Kunth, Woodsia mollis (Kaulf.) J. Sm.), estolones (Houstonia serpyllacea (Schltdl.) C.L. Sm. ex Greenm., Phacelia platycarpa (Cav.) Spreng. y Trifolium amabile Kunth,) y rosetas ( algunas son Echeandia matudae Cruden, Eryngium carlinae F. Delaroche, Lepechinia caulescens (Ortega) Epling y Weldenia candida Schult.), que están creciendo muy junto al suelo. El herbáceo superior por especies anuales (Bidens chiapensis Brandegee, B. ostruthioides (DC.) Sch. Bip., B. triplinervia Kunth, Lopezia hirsuta Jacq., Salvia cinnabarina M. Martens \& Galeotti, Senecio callosus Sch. Bip., S. godmanii Hemsl., S. warszewiczii A. Braun \& Bouché, Sigesbeckia jorullensis Kunt, Stevia incognita Grashoff, entre las más dominantes) y semiperennes que sobreviven por sus órganos subterráneos de un año a otro (se pueden mencionar a Arracacia atropurpurea (Lehm.) Benth. \& Hook. f. ex Hemsl., A. xanthorrhiza Bancr., Calamagrostis vulcanica Swallen, Castilleja integrifolia var. alpigena L.O. Wiliams, Daucus montanus Humb. \& Bonpl. ex Spreng., Lamourouxia dependens Benth., L. xalapensis Kunt, Muhlenbergia macroura (Kunth) Hitchc., Piptochaetium fimbriatum (Kunth) Hitchc. y Ranunculus geoides Kunt ex DC). El estrato de arbustos por semiperennes y perennes con adaptaciones para sobrevivir en la época seca y de heladas (entre las más abundantes están Acaena elongata L., Baccharis vaccinioides Kunth, Buddleia megalocephala Donn.Sm., Comarostaphylis arbutoides Lindl., Dahlia imperialis Roezl ex Ortgies, Eupatorium sp. L., Fuchsia splendens Zucc., F. striolata Lundell, Gaultheria sp. L., Lupinus ehrenbergii Schltdl., Roldana heterogama (Benth.) H. Rob. \& Brettell., Rumex acetosella
L., Rubus trilobus Moc. \& Sessé ex Ser., Salvia lavanduloides Kunth, Stevia polycephala Bertol., Symphoricarpos microphyllus Kunth, Verbesina apleura S.F. Blake, V. hypoglauca Sch. Bip. ex Klatt y Polystichum speciosissimum (A. Braun ex Kunze) Copel.). En el de árboles (Abies guatemalensis Rehder, Alnus acuminata Kunt, Neocupressus lusitanica (Mill.) de Laub., Pinus ayacahuite C. Ehrenb. ex Schltdl., P. rudis Endl., Prunus serotina Ehrh. y Quercus skinneri Benth.).

Distribución geográfica: Las especies se catalogaron en seis grupos de distribución geográfica (Fig. 1). El grupo 1 contiene 80, es el más numeroso, está conformado por aquellas que se distribuyen del centro de México hasta Centroamérica. El grupo 2 tiene 25 y lo constituye las que tienen una distribución de México a Sudamérica. Estos dos grupos contienen el $88 \%$ de la flora que crecen en los bosques de abeto del área de estudio. El grupo 3 está conformado por cinco con distribución desde Estados Unidos de Norteamérica hasta Centroamérica, el grupo 4 incluye cinco que tienen una distribución en América. El grupo 5 contiene dos de amplia distribución, Rumex acetosella L. y Neocupressus lusitanica (Mill.) de Laub., que a pesar de tener un origen mesoamericano se les encuentra en Europa y Asia, continentes a los cuales fueron introducidas. El grupo 6 contiene dos especies, que muestra que el componente Sudamericano es escaso en los bosques de Abies.

\section{DISCUSIÓN}

De acuerdo con Bermúdez \& Sánchez (2000), la parte montana occidental de Guatemala ha sido objeto de muchas recolectas botánicas, que están almacenadas en los principales herbarios del país. Sin embargo, son escasas las publicaciones científicas basadas en esos especímenes, por lo que hay poco conocimiento sistematizado de inventarios florísticos y estudios ecológicos. El presente estudio contribuye a superar en parte el vacío de documentación, al publicar la lista de especies encontradas en 


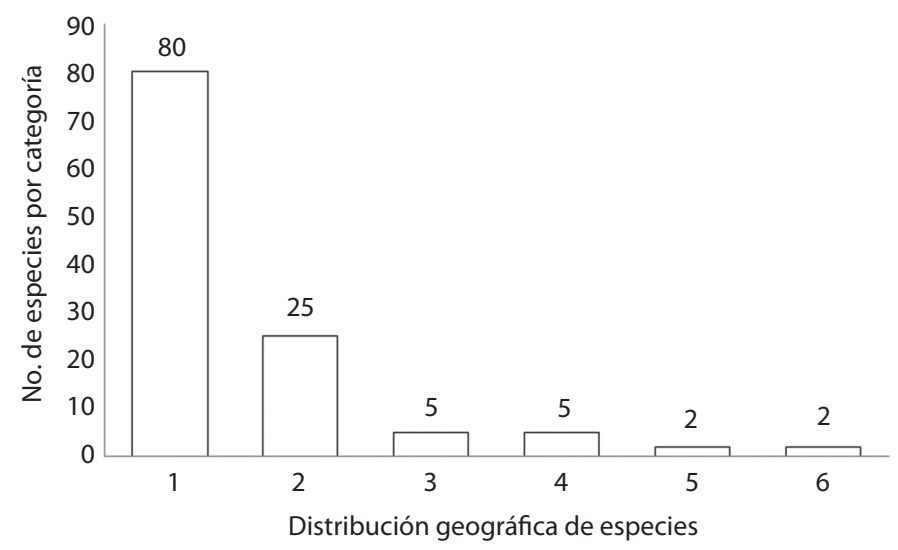

Fig. 1. Distribución geográfica de la flora de bosques de abeto (Abies guatemalensis) de la parte alta de San Marcos, Guatemala. 1: Centro de México hasta Centroamérica, 2: México hasta Sudamérica, 3: Estados Unidos de Norteamérica hasta Centroamérica, 4: América; 5: Amplia distribución y 6: Guatemala hasta Sudamérica.

Fig. 1. Geographical distribution of flora of fir forests (Abies guatemalensis) of the upper San Marcos, Guatemala. 1: Central Mexico to Central America, 2: Mexico to South America, 3: United. States of America to Central America, 4: America, 5: Wide distribution and 6: Guatemala to South America.

los bosques de abeto de la parte occidental de país, y aumentar el conocimiento del ambiente donde crece $A$. guatemalensis que es una especie protegida para Guatemala (CONAP 2009) y estas acciones contribuyen a su conservación.

En los bosques estudiados el $49 \%$ de las especies se concentró en seis familias botánicas, situación similar fue encontrada por Sánchez-González et al. (2005) en bosques de abeto de México. Por otra parte hay $75(81 \%)$ de los 92 géneros registrados que contienen solo una especie, lo que refuerza lo afirmado por Galindo et al. (2003), en el sentido de que en los ecosistemas templados $\mathrm{y}$ fríos hay una alta heterogeneidad en especies. En su mayoría son grupos taxonómicos con mecanismos morfológicos, anatómicos y fisiológicos que les permiten adaptarse a las condiciones de temperatura, en especial las heladas que se presentan de noviembre a marzo (Cuevas-Guzmán et al. 2011).

En otros trabajos florísticos del área se ha obviado considerar la división Briophyta en la lista de especies (Veliz 1998, Veliz et al. 2001, Viñals et al. 2001). En el presente estudio, se incluyó por la importancias ecológica de los musgos en estos ecosistemas, en especial en el proceso hídrico (Rams 2008, Tobón 2009), se ha encontrado que hay un vacío de información, sobre su taxonomía, biogeografía y ecología en el país (Véliz 2008), que visualizan la necesidad de llevar a cabo estudios en este interesante tema (Glime 2007).

Las 119 especies consignadas en este estudio para las áreas de bosque de abeto, puede considerarse como alta riqueza florística y apoya la propuesta de conservación a partir de parches de bosque (Corrêa et al. 2001). Para reforzar el argumento de la alta riqueza, puede mencionarse, que Veliz et al. (2001) en su estudio florístico de la porción Tajumulco-Tacana, en una amplitud altitudinal de $3000-3400 \mathrm{~m}$, la cual comprende la región de estudio, registraron 141 especies en un muestreo que abarcó áreas de cultivo, terrenos abandonados, zonas de pastoreo, bosques y otros. De tal forma que al hacer una relación entre ambos trabajos, los parches de abeto estudiados incluyen el $84 \%$ de la flora de la porción estudiada de TajumulcoTacana. A pesar que debe reconocerse que en los parches de bosque es muy probable la extinción de especies (MacArthur \& Wilson 
1967), también debe considerarse que en las condiciones del área de estudio, es lo queda para propiciar la conservación. La alta cantidad de especies presentes en los remanentes de bosque en relación a otras áreas degradadas, se debe en parte al microclima que se genera (Echeverry \& Rodríguez 2006) y que promueve la existencia y sobrevivencia de estas en los bordes y dentro del mismo bosque, lo cual es importante ya que convierte a estas áreas en acervos de diversidad e instrumentos para la conservación (Cayuela 2006).

A pesar de haber 94 bosques de A. guatemalensis, en forma de parches en Guatemala, distribuidos en la sierra de los Cuchumatanes, cadena volcánica (San Marcos, Quetzaltenango, Totonicapán y Sololá), Jalapa y Sierra de la Minas, y de ser el abeto una especie protegida (CONAP 2008), hay poca información documentada de la flora de esos lugares, esto impide hacer comparaciones florísticas de esas áreas con los resultados del presente estudio que serían de utilidad para contribuir en el conocimiento de la biodiversidad y fitogeografía local. Nuevamente se enfatiza en la importancia, que estudios similares al presente, se realicen en los otros bosques de abeto para propiciar un mejor conocimiento de este tipo de ecosistema que ayude a su adecuada conservación.

La información obtenida, permite tener una mejor representación del comportamiento de la composición de la flora de los bosques de abeto estudiados. Por una parte se puede notar que hay afinidad florística en los bosques de abeto de la cordillera volcánica de Guatemala al comparar con la información proporcionada por González \& Castañeda (1983) y Veliz et al. (2001). En tanto, que con relación a la sierra de los Cuchumatanes, ubicada hacia el norte, hay menor número de especies en el área del presente estudio (Veliz 1998), que se explica por ser esa región de alta diversidad en Guatemala, por su origen geológico más antiguo y por la influencia de la flora Holártica (Castañeda 2008). Comparado con la flora de la zona alta de Totonicapán, ubicada hacia el sur, la riqueza encontrada en esta investigación es mayor (Veliz et al. 2001). En el caso de los bosques, similares a los del presente estudio, ubicados en el centro y sur de México, hay un reconocimiento de Islebe et al. (1994), Velázquez \& Islebe (1995) sobre la similitud de la flora de las partes altas del occidente de Guatemala con las del sur y centro de México. De la misma manera, la composición de especies en los bosques de abeto para las partes mencionadas de ambos países también guarda afinidad, en especial a nivel de familias y géneros (Madrigal 1967, Sánchez et al. 1991, Mendoza 2003, Sánchez-González et al. 2005, Sánchez et al. 2006). Aunque tiene que reconocerse, que pesar de que se tenga semejanza entre los bosques del área de estudio y sus homólogos del centro y sur de México, debe considerarse que hay particularidades, influenciadas por el suelo, microclima y latitud que deben tomarse en cuenta al momento del manejo y conservación de estas áreas (Sanchez-González et al. 2005).

Hay una marcada dominancia de la flora que tiene su origen de México a Centroamérica (67\%), esto muestra la influencia del origen Holártico en los bosque de abeto (Castañeda 2008) y apoyan la idea de que posiblemente estas especies han convivido desde hace mucho tiempo en los bosques que se conformaron hacia el sur de México y occidente de Guatemala, en la última glaciación y sus periodos interglaciares (Arroyo-Cabrales et al. 2008), en los que también A. guatemalensis se desplazó en su distribución hacia el sur de América. (Jaramillo-Correa et al. 2008). Rzedowski (1991) para explicar el origen de la flora fanerógama de México propone el área fitogeográfica llamada Megaméxico 2 que comprende del centro de México al norte de Costa Rica (Mesoamérica), que contiene el $64 \%$ de especies endémicas, lo cual concuerda con el grupo 1 propuesto en este estudio, y confirma nuevamente que la fitogeografía de la flora de los bosques de Abies del centro de México a Guatemala tienen mucha similitud en cuanto compartir una historia geológica similar (Coates 2003) además de una composición y estructura florística análoga como lo han señalado Velázquez \& Islebe (1995). 
Las áreas de bosque de $A$. guatemalensis son objeto de protección por parte de la institución del estado dedicada a la conservación, que también es la encargada de coordinar las acciones de la estrategia nacional del abeto. Dentro de ellas está la restauración ecológica, con el fin de aumentar el tamaño actual de estos bosques (CONAP 2010). En este sentido, la información obtenida en el presente estudio será de beneficio para diversas actividades, una de ellas es identificar y utilizar las especies arbustivas que puedan funcionar como nodrizas (Padilla \& Pugnaire 2006) en el proceso de regeneración natural y establecimiento de árboles.

\section{AGRADECIMIENTOS}

El autor agradece al Programa Doctoral en Ciencias Naturales para el Desarrollo (DOCINADE) sede Universidad Estatal a Distancia de Costa Rica y a la Secretaria Nacional de Ciencia y Tecnología (SENACYT) de Guatemala, que por medio del Fondo para la Ciencia y Tecnología (FODECYT) financió el proyecto FODECYT 055-2009, del cual esta información forma parte. A David Mendieta, herbario AGUAT por la revisión y observaciones al manuscrito.

\section{RESUMEN}

Hay una carencia de información detallada sobre la composición y estructura de las comunidades montanas guatemaltecas. El objetivo del estudio fue contribuir al conocimiento de la flora de bosques de abeto (Abies guatemalensis), para esto se hizo el levantamiento florístico en bosques de abeto del occidente de Guatemala. Se encontraron 119 especies, 92 géneros, 50 familias en cuatro divisiones. Las familias más numerosas fueron: Asteraceae, Poaceae, Rosaceae, Lamiaceae, Apiaceae y Solanaceae y los géneros más abundantes Salvia, Alchemilla y Bidens. Las especies se ubicaron en cuatro estratos, 33 en el herbáceo inferior, 49 en el herbáceo superior, 30 en arbustos y siete en árboles. Se hace énfasis en la contribución del estudio al conocimiento de la flora de bosques de $\mathrm{A}$. guatemalensis y la necesidad de otros similares en los demás bosques de esta especie, que sirva para fomentar su conocimiento y conservación. Se consideraron seis grupos de distribución geográfica, el principal es del centro de México a Centroamérica con $67 \%$ de especies. Se realiza una comparación fitogeográfica y de composición florística, con otras áreas de Abies de Guatemala y México. Se propone que a pesar de haber familias y géneros comunes, que proporcionan la estructura general entre los bosques de abeto, se deben considerar las particularidades florísticas de cada área, en el manejo y conservación influidas por suelo, latitud y microclima.

Palabras clave: flora, conservación, riqueza florística, abeto, Abies guatemalensis, distribución geográfica.

\section{REFERENCIAS}

Arroyo-Cabrales, J., A.L. Carreño, S. Lozano-García \& M. Montellano-Ballesteros. 2008. La diversidad en el pasado, p. 227-262. In Capital natural de México, vol. I: Conocimiento actual de la biodiversidad. Conabio, México D.F., México.

Ávila, C.H. \& L. López. 2001. Distribución y análisis estructural de Abies hicklii (Flous \& Gaussen) en México. Interciencia. 26: 244-251.

Bermúdez, M. \& G.J. Sánchez (eds.). 2000. Identificación de vacíos de información botánica en Centroamérica. WWF Centroamérica, Museo Nacional de Costa Rica y Red de Herbarios de Mesoamérica y el Caribe, San José, Costa Rica.

Bertram, E.B. 1949. Mosses of Guatemala. Field. Bot. 25: $1-458$.

Bouroncle, C. 2008. Efectos de la fragmentación en la ecología reproductiva de especies y grupos funcionales del bosque húmedo tropical de la zona atlántica de Costa Rica. Tesis de Maestría, CATIE, Turrialba, Costa Rica.

Castañeda, C.A. 2008. Biodiversidad de Guatemala, en relación con su historia geológica y biogeografía, p. 181-229. In Consejo Nacional de Áreas Protegidas. Guatemala y su biodiversidad: un enfoque histórico, cultural, biológico y económico. Consejo Nacional de Áreas Protegidas, Guatemala, Guatemala.

Cayuela, L. 2006. Deforestación y fragmentación de bosques tropicales montanos en los altos de Chiapas, México. Efectos sobre la diversidad de árboles. Ecosistemas 15: 192-198.

Coates, A.G. 2003. La forja de Centroamérica, p. 1-40. In A.G. Coates (ed.). Paseo pantera: una historia de la naturaleza y cultura de Centroamérica. Instituto Smithsoniano, Washington, EE.UU.

CONAP. 2008. Diagnóstico del contexto institucional, ambiental y forestal para la conservación y fomento de los bosques naturales y plantaciones de pinabete (Abies guatemalensis Rehder). Consejo Nacional de Áreas Protegidas, Guatemala, Guatemala.

CONAP. 2009. Lista de especies amenazadas de Guatemala (LEA) y Listado de especies de flora y fauna 
CITES de Guatemala. Consejo Nacional de Áreas Protegidas, Guatemala, Guatemala.

CONAP. 2010. Estrategia nacional para la conservación del pinabete para el periodo 2008-2017. Documento técnico No. 80. Consejo Nacional de Áreas Protegidas, Guatemala, Guatemala.

Corrêa, A.P., B. Finegan \& C. Harvey. 2001. Evaluación y diseño de un paisaje fragmentado para la conservación de biodiversidad. Rev. For. Centroamericana 34: 35-41.

Cuevas-Guzmán, R., E.A. Cisneros-Lepe, E.J. JardelPeláez, E.V. Sánchez-Rodríguez, L. Guzmán-Hernández, N.M. Núñez-López \& C. Rodríguez-Guerrero. 2011. Análisis estructural y de diversidad en los bosques de Abies de Jalisco, México. Rev. Mex. Biodiv. 82: 1219-1233.

Echeverría, M.A. \& J.M. Rodríguez. 2006. Análisis de un paisaje fragmentado como herramienta para la conservación de la biodiversidad en áreas de bosque seco y subhúmedo tropical en el municipio de Pereira, Risaralda, Colombia. Sc. et Tech. 30: 405-410.

Ejtehadi, H., T. Amini \& H. Zare. 2005. Importance of vegetation studies in conservation of wildlife: a case study in Miankaleh wildlife refuge, Mazandaran province, Iran. Environ. Sci. 9: 53-58.

FAO. 2007. Base referencial mundial del recurso suelo. Informes sobre recursos mundiales de suelos No. 103. IUSS grupo de trabajo WRB. Organización de Naciones Unidas para la Alimentación y la Agricultura, Roma, Italia.

Galindo, R., J. Betancur \& J. Cadena. 2003. Estructura y composición florística de cuatro bosques andinos del santuario de flora y fauna Guanentá-alto río Fonce, cordillera oriental colombiana. Caldasia 25: 313-335.

Glime, J.M. 2007. Economic and Ethnic Uses of Bryophytes, p. 14-41. In: America Editorial Committee. Flora of North America Vol. 27 Briophytas: Mosses Oxford University, Nueva York, EE.UU.

González, J.H. \& C. Castañeda. 1983. Las comunidades de pinabete (Abies guatemalensis Rehder) en Guatemala. Tikalia 1: 4-37.

Heywood, V. 2004. Modern approaches to floristics and their impact on the region of SW Asia. Turk. J. Bot. 28: 7-16

Holdridge, L.R. 1978. Ecología basada en zonas de vida. Instituto Interamericano de Cooperación para la Agricultura, San José, Costa Rica.

INSIVUMEH. 2003. Atlas hidrológico de Guatemala. Instituto Nacional de Vulcanología, Meteorología e Hidrología (Consultado: 25 enero 2012, www.insivumeh.gob.gt/hidrologia/ATLAS_HIDROMETEOROLOGICO/Atlas Clima.html).

Islebe, G.A., A.M. Cleef \& A. Velázquez. 1994. Especies leñosas de la sierra de los Cuchumatanes y de la cadena montañosa, Guatemala. Acta Bot. Mex. 29: 83-92.

Jaramillo-Correa, J.P., E. Aguirre-Planter, D.P. Khasa, L.E. Eguiarte, D. Piñero, G.R. Furnier \& J. Bousquet. 2008. Ancestry and divergence of subtropical montane forest isolates: molecular biogeography of the genus Abies (Pinaceae) in Southern México and Guatemala. Mol. Ecol. 17: 2476-2490.

Knapp, S., G. Davidse \& M. Sousa. 2001. Proyectos florísticos hoy y mañana: su importancia en la sistemática y la conservación, p 331-358. In H.M. Hernández, A.N. García, F. Álvarez \& M. Ulloa (eds.). Enfoques contemporáneos para el estudio de la biodiversidad. Fondo de Cultura Económica, México D.F., México.

López-Sandoval, J.A., E. Koch, L.M. Vásquez-García, J. Munguía-Lino \& E.J. Morales-Rosales. 2010. Estudio florístico de la parte central de la barranca Nenetzingo, municipio de Ixtapan de la Sal, estado de México. Polibotánica 30: 9-33.

Luna-Vega, I. 2008. Aplicaciones de la biogeografía histórica a la distribución de las plantas mexicanas. Rev. Mex. Biodiv. 79: 217-241.

MacArthur, R.H. \& E.O. Wilson. 1967. The theory of island biogeography. Princeton, Nueva Jersey, EE.UU.

Madrigal, X. 1967. Contribuciones al conocimiento de la ecología de los bosques de oyamel (Abies religiosa (H.B.K.) Schl. \& Cham. en el valle de México. Boletín Técnico No. 18. Instituto Nacional de Investigaciones Forestales, México D.F., México.

Melgar, W. 2003. Estado de la diversidad biológica de árboles y bosques de Guatemala. Documentos de Trabajo FGR/53S. Servicio de Desarrollo de Recursos Forestales, Dirección de Recursos Forestales, FAO, Roma, Italia.

Mendoza, E. 2003. Clasificación y ordenación de los bosques de Abies religiosa (H.B.K.) Cham. \& Schltdl. en la Faja Volcánica Transmexicana. Tesis de Maestría, Universidad Autónoma de Chapingo, Chapingo, México.

Morrone, J. 2001. Biogeografía de América Latina y el Caribe. Vol. 3. M\&T-Manuales \& Tesis SEA, Zaragoza, España.

Olguín, K. 2008. Estudio florístico preliminar en la región forestal Chignahuapan-Zacatlán, Puebla. Tesis de Licenciatura, Universidad Autónoma de Chapingo, Chapingo, México.

Padilla, F.M. \& F.I. Pugnaire 2006. The role of nurse plants in the restoration of degraded environments. Ecol. Environ. 4: 196-202.

Ponce, A., I. Luna, O. Alcántara \& C.A. Ruíz. 2006. Florística del bosque mesófilo de montaña de Monte Grande, Lolatla, Hidalgo, México. An. Inst. Biol. 77: 177-190. 
Prima, A. \& G. Alfonso. 2002. La importancia de las prospecciones florísticas en biología de conservación. Una experiencia en el árido del centro-oeste de Argentina. Ecosistemas 3 (Consultado: 10 mayo 2012, www. aeet.org/ecosistemas/investigacion4.htm).

Rams, S. 2008. El papel ecológico de los briófitos. Eubacteria 20: 15-18. (Consultado: 15 junio 2011, http:// www.um.es/eubacteria/Eubacteria.html).

Rasmussen, K.K., U. Strandby \& J. Kollmann. 2010. High genetic diversity within but limited differentiation among populations of the vulnerable Guatemalan fir. J. Trop. Forest Sc. 22: 247-259.

Rzedowski, J. 1991. Diversidad y orígenes de la flora fanerógamica de México. Acta Bot. Mex. 14: 3-21.

Sánchez-González, A., L. López-Mata \& D. GranadosSánchez. 2005. Semejanza florística entre los bosques de Abies religiosa (H.B.K.) Cham. \& Schltdl. de la Faja Volcánica Transmexicana. Bol. Inst. Geogr. 56: 62-76.

Sánchez, A., L. López \& H. Vibrans. 2006. Composición y patrones de distribución de la flora del bosque de oyamel del cerro Tlaloc, México. Bol. Soc. Bot. Mex. 79: 67-78.

Sánchez, L.R., M. Pineda \& M.A. Hernández. 1991. Distribución y estructura de la población de Abies religiosa (H.B.K.) Schl. \& Cham., en el Cofre de Perote, estado de Veracruz, México. Acta Bot. Mex. 16: 45-55.

Sánchez, V. \& N. Grados. 2007. Estudio florístico y ambiental del bosque relicto Mijal en la provincia de Morropón, Piura. Arnaldoa 14: 259-268.

Shuster, J.C. \& S.B. Bonis. 2008. Biodiversidad de Guatemala, en relación con su historia geológica y biogeografía, p. 1-34. In Consejo Nacional de Áreas Protegidas. Guatemala y su biodiversidad: un enfoque histórico, cultural, biológico y económico. Consejo Nacional de Áreas Protegidas, Guatemala.

Simmons, S., M.J. Tarano \& J.H. Pinto. 1959. Clasificación y reconocimiento de los suelos de la república de Guatemala. José de Pineda Ibarra, Guatemala.

Simonetti, J.A. 2006. Conservación de biodiversidad en ambientes fragmentados: el caso del bosque maulino, p. 213-232. In A.A. Grez, J.A. Samotti \& R.O. Bustamante (eds.). Biodiversidad en ambientes fragmentados de Chile: patrones y procesos a diferentes escalas. Universidad de Chile, Santiago, Chile.
Standley, P. \& J. Steyermark. 1947-1977. Flora of Guatemala. Fieldiana Botany 24.

Stevens, W.D., C. Uloa, A. Pool \& O. Montiel (eds.). 2001. Flora de Nicaragua. Vo. 85. Missouri Botanical Garden, San Luis Misuri, EE.UU.

Stevenson, P.R. \& M.E. Rodríguez. 2008. Determinantes de la composición florística y efectos de borde en un fragmento de bosque en la Guaviare, Amazonía colombiana. Rev. Colombiana For. 11: 15-17.

Stolze, R.G. 1981. Ferns and fern allies of Guatemala. Field. Bot. 6: 1-548.

Strandby, U., K.I. Christensen \& M. Sørensen. 2009. A morphometric study of the Abies religiosa-hickeliiguatemalensis complex (Pinaceae) in Guatemala and Mexico. Plant. Syst. Evol. 280: 59-76.

Tobón, C. 2009. Los bosques andinos y el agua. Serie investigación y sistematización No. 4. Programa Regional ECOBONA-INTERCOOPERATION, Quito, Ecuador.

Toledo, M., J. Salick, B. Loiselle \& P. Jørgensen. 2005. Composición florística y usos de bosques secundarios en la provincia Guarayos, Santa Cruz, Bolivia. Rev. Bol. Ecol. 18: 1-16.

Velázquez, A. \& G.A. Islebe. 1995. Comparación fitogeográfica entre las montañas del centro de México y Guatemala. Caldasia 17: 501-508.

Véliz, M. 1998. Composición florística de la meseta alta de la sierra de los Cuchumatanes, Huehuetenango, Guatemala. Ciencia y Tecnología 4: 11-34.

Véliz, M. 2008. Diversidad florística de Guatemala, p. 261-298. In Consejo Nacional de Áreas Protegidas. Guatemala y su biodiversidad: un enfoque histórico, cultural, biológico y económico. Consejo Nacional de Áreas Protegidas, Guatemala.

Véliz, M., N. Gallardo, M. Vásquez \& R. Luarca. 2001. La vegetación montana de Guatemala. Ciencia y Tecnología 6: 3-61.

Viñals, J., H. Paniagua, H. Barillas \& M. Véliz. 2001. Estudio de la composición florística de las cimas de los volcanes, Agua, Acatenango, Fuego, Atitlán, Zunil, Santo Tomás (Pecúl), Santa María, Tajumulco y Tacana, en la república de Guatemala. Ciencia y Tecnología 6: 3-47.

Zamora, P. 2003. Contribución al estudio florístico y descripción de la vegetación del municipio de Tenabo, Campeche, México. Polibotanica 15: 1-40. 


\section{APÉNDICE 1}

Especies que crecen en los bosques de Abies guatemalensis y sus alrededores y el estrato de vegetación en que se clasificó

\section{APPENDIX 1}

Species list growing in the fir forests of Abies guatemalensis and its surroundings and the vegetation strata where is classified

\begin{tabular}{|c|c|c|c|c|}
\hline Familia & Nombre científico & Estrato & $\begin{array}{l}\text { Distribución } \\
\text { geográfica }\end{array}$ & $\begin{array}{l}\text { Colector y } \\
\text { número* }\end{array}$ \\
\hline División & Briophyta & & & \\
\hline Hypnaceae & Hypnum amabile (Mitt.) Hampe & HI & 2 & FRPM675A \\
\hline Hypnaceae & Mitthenothamnium reptans (Hedw.) Card. & HI & 2 & FRPM 674 \\
\hline Hypnaceae & $\begin{array}{l}\text { Vesicularia vesicularis (Schwägr.) } \\
\text { Broth. var. portoricensis (Brid.) Buck. }\end{array}$ & $\mathrm{HI}$ & 2 & FRPM 652 \\
\hline Hypopterygiaceae & Hypopterygium tamariscinum (Hedw.) Brid. & $\mathrm{HI}$ & 4 & FRPM 675 \\
\hline Pilotrichaceae & Cyclodictyon albicans (Hedw.) Kuntze & $\mathrm{HI}$ & 2 & FRPM 595 \\
\hline Sematophyllaceae & Sematophyllum galipense (C. Mull.) Mitt. & $\mathrm{HI}$ & 2 & FRPM 674A \\
\hline División & Pteridophyta & & & \\
\hline Alpleniaceae & Asplenium costaneum Schltdl. \& Cham. & HI & 2 & FRPM 15 \\
\hline Dryopteridaceae & Polystichum speciosissimum (A. Braun ex Kunze) Copel. & AR & 1 & FRPM 52 \\
\hline Dryopteridaceae & Polystichum fournieri A.R. Sm. & AR & 1 & FRPM 523 \\
\hline Polypodiaceae & Campyloneurum xalapense Fée & $\mathrm{HI}$ & 1 & S.N. \\
\hline Pteridaceae & Adianthum andicola Liebm. & $\mathrm{HI}$ & 2 & S.N. \\
\hline Pteridaceae & Cheilanthes sp. Sw. & $\mathrm{HI}$ & 2 & FRPM 58 \\
\hline Woodsiaceae & Woodsia mollis (Kaulf.) J. Sm. & $\mathrm{HI}$ & 1 & FRPM 584 \\
\hline División & Pinophyta & & & \\
\hline Cupressaceae & Neocupressus lusitanica (Mill.) de Laub. & $\mathrm{AB}$ & 6 & FRPM 569 \\
\hline Pinaceae & Abies guatemalensis Rehder & $\mathrm{AB}$ & 1 & FRPM 566 \\
\hline Pinaceae & Pinus ayacahuite C. Ehrenb. ex Schltdl. & $\mathrm{AB}$ & 1 & GIFC 95 \\
\hline Pinaceae & Pinus rudis Endl. & $\mathrm{AB}$ & 1 & GIFC 96 \\
\hline División & Magnoliophyta & & & \\
\hline Clase & Liliopsida & & & \\
\hline Alstroemeriaceae & Bomarea hirtella (Kunth) Herb. & HS & 1 & FRPM 63 \\
\hline Asparagaceae & Echeandia matudae Cruden & HI & 1 & S.N. \\
\hline Asparagaceae & Smilacina scilloidea M. Martens \& Galeotti & $\mathrm{HI}$ & 1 & FRPM 531 \\
\hline Cyperaceae & Carex donnell-smithii L.H. Bailey & HS & 1 & FRPM 43 \\
\hline Iridaceae & Tigridia immaculata (Herb.) Ravenna & HS & 1 & FRPM 621 \\
\hline Juncaceae & Luzula carisina E. Meyer & HS & 1 & FRPM 623 \\
\hline Poaceae & Agrostis vesca Swallen & HS & 1 & FRPM 42 \\
\hline Poaceae & Brachypodium mexicanum (Roem. \& Schult.) Link & HI & 2 & FRPM 8 \\
\hline Poaceae & Bromus exaltatus Bernh. & $\mathrm{HI}$ & 1 & FRPM 700 \\
\hline Poaceae & Bromus laciniatus Beal & HS & 1 & FRPM 626 \\
\hline Poaceae & Calamagrostis vulcanica Swallen & HS & 1 & FRPM 565 \\
\hline Poaceae & Eragrostis sp. Wolf & HS & 2 & FRPM 628 \\
\hline Poaceae & Muhlenbergia macroura (Kunth) Hitchc. & HS & 1 & FRPM 53 \\
\hline Poaceae & Piptochaetium fimbriatum (Kunth) Hitchc. & HS & 3 & FRPM 622 \\
\hline Poaceae & Piptochaetium sp. J. Presl & HS & 3 & S.N. \\
\hline Clase & Magnoliopsida & & & \\
\hline Amaranthaceae & Celosia paniculata $\mathrm{L}$. & $\mathrm{HI}$ & 4 & FRPM 582 \\
\hline
\end{tabular}




\begin{tabular}{|c|c|c|c|c|}
\hline Familia & Nombre científico & Estrato & $\begin{array}{l}\text { Distribución } \\
\text { geográfica }\end{array}$ & $\begin{array}{l}\text { Colector y } \\
\text { número* }\end{array}$ \\
\hline Apiaceae & $\begin{array}{l}\text { Arracacia atropurpurea (Lehm.) } \\
\text { Benth. \& Hook. f. ex Hemsl. }\end{array}$ & HS & 2 & EMFS 10 \\
\hline Apiaceae & Arracacia xanthorrhiza Bancr. & HS & 5 & FRPM 618 \\
\hline Apiaceae & Daucus montanus Humb. \& Bonpl. ex Spreng. & HS & 2 & FRPM 612 \\
\hline Apiaceae & Eryngium carlinae F. Delaroche & $\mathrm{HI}$ & 1 & FRPM 615 \\
\hline Apiaceae & Eryngium cymosum F. Dolaroche & HS & 1 & FRPM 28 \\
\hline Araliaceae & Hydrocotile mexicana Schltdl. \& Cham. & $\mathrm{HI}$ & 1 & EMFS 13 \\
\hline Araliaceae & Oreopanax echinops (Cham. \& Schltdl.) Decne. \& Planch. & $\mathrm{AR}$ & 1 & EMFS 6 \\
\hline Asteraceae & Baccharis vaccinioides Kunth & $\mathrm{AR}$ & 1 & FRPM 50 \\
\hline Asteraceae & Bidens chiapensis Brandegee & HS & 1 & FRPM 54 \\
\hline Asteraceae & Bidens ostruthioides (DC.) Sch. Bip. & HS & 1 & FRPM 607 \\
\hline Asteraceae & Bidens sp. L. & HS & 1 & FRPM 608 \\
\hline Asteraceae & Bidens triplinervia Kunth & HS & 2 & FRPM 631 \\
\hline Asteraceae & Cirsium radians Benth. & HS & 1 & FRPM 48 \\
\hline Asteraceae & Dahlia imperialis Roezl ex Ortgies & $\mathrm{AR}$ & 2 & S.N. \\
\hline Asteraceae & Erigeron karvinskianus DC. & HI & 1 & FRPM \\
\hline Asteraceae & Eupatorium sp. L. & $\mathrm{AR}$ & 1 & FRPM 38 \\
\hline Asteraceae & Fleishmannia sp. Sch. Bip. & AR & 1 & FRPM 1002 \\
\hline Asteraceae & Gamochaeta standleyi (Steyerm.) G.L. Nesom & HS & 1 & S.N. \\
\hline Asteraceae & Gnaphalium liebmannii Sch. Bip. ex Klatt & HS & 1 & FRPM 616 \\
\hline Asteraceae & Montanoa pteropoda S.F. Blake & $\mathrm{AR}$ & 1 & FRPM 701 \\
\hline Asteraceae & Roldana heterogama (Benth.) H.Rob. \& Brettell & AR & 1 & EMFS 7 \\
\hline Asteraceae & Senecio callosus Sch. Bip. & HS & 1 & FRPM 57 \\
\hline Asteraceae & Senecio godmanii Hemsl. & HS & 1 & EMFS 5 \\
\hline Asteraceae & Senecio warszewiczii A. Braun \& Bouché & HS & 1 & FRPM 36 \\
\hline Asteraceae & Sigesbeckia jorullensis Kunt & HS & 2 & FRPM 35 \\
\hline Asteraceae & Stevia incognita Grashoff & HS & 2 & EMFS 18 \\
\hline Asteraceae & Stevia lucida var. oaxacana (DC.) Grashoff & HS & 1 & FRPM 51 \\
\hline Asteraceae & Stevia polycephala Bertol. & $\mathrm{AR}$ & 1 & FRPM 12 \\
\hline Asteraceae & Tagetes foetidissima DC. & HS & 1 & FRPM 37 \\
\hline Asteraceae & Verbesina apleura S.F. Blake & $\mathrm{AR}$ & 1 & FRPM 672 \\
\hline Asteraceae & Verbesina hypoglauca Sch. Bip. ex Klatt & $\mathrm{AR}$ & 1 & FRPM 13 \\
\hline Betulaceae & Alnus acuminata Kunt & $\mathrm{AB}$ & 2 & FRPM 587 \\
\hline Boraginaceae & Hackelia skutchii I.M. Johnst. & HS & 5 & FRPM 594 \\
\hline Campanulaceae & Lobelia umbellifera McVaugh & HS & 1 & EMFS 19 \\
\hline Caprifoliaceae & Symphoricarpos microphyllus Kunth & $\mathrm{AR}$ & 3 & FRPM 57 \\
\hline Caryophyllaceae & $\begin{array}{l}\text { Cerastium brachypodum (Engelm. ex A. Gray) } \\
\text { B.L Rob. ex Britton }\end{array}$ & HS & 3 & FRPM 632 \\
\hline Commelinaceae & Weldenia candida Schult. f. & $\mathrm{HI}$ & 1 & FRPM 643 \\
\hline Ericaceae & Comarostaphylis arbutoides Lindl. & $\mathrm{AR}$ & 1 & EMFS 21 \\
\hline Ericaceae & Gaultheria sp. L. & AR & 1 & FRPM 628 \\
\hline Euphorbiaceae & Euphorbia orizabae Boiss. & HS & 1 & FRPM 52 \\
\hline Fabaceae & Lupinus ehrenbergii Schltdl. & $\mathrm{AR}$ & 1 & FRPM 29 \\
\hline Fabaceae & Trifolium amabile Kunth & $\mathrm{HI}$ & 4 & FRPM 624 \\
\hline Fagaceae & Quercus skinneri Benth. & $\mathrm{AB}$ & 1 & FRPM 710 \\
\hline Gentianaceae & Halenia decumbens Benth. & HS & 1 & FRPM 614 \\
\hline Geraniaceae & Geranium alpicola Loes. & $\mathrm{HI}$ & 1 & EMFS 19 \\
\hline Geraniaceae & Geranium repens H.E. Moore & HI & 1 & FRPM 601 \\
\hline
\end{tabular}




\begin{tabular}{|c|c|c|c|c|}
\hline Familia & Nombre científico & Estrato & $\begin{array}{l}\text { Distribución } \\
\text { geográfica }\end{array}$ & $\begin{array}{l}\text { Colector y } \\
\text { número* }\end{array}$ \\
\hline Hydrophyllaceae & Phacelia platycarpa (Cav.) Spreng. & $\mathrm{HI}$ & 1 & FRPM 1020 \\
\hline Lamiaceae & Lepechinia caulescens (Ortega) Epling & $\mathrm{HI}$ & 1 & FRPM 572 \\
\hline Lamiaceae & Salvia cinnabarina $\mathrm{M}$. Martens \& Galeotti & HS & 1 & FRPM 678 \\
\hline Lamiaceae & Salvia excelsa Benth. & HS & 1 & FRPM 1001 \\
\hline Lamiaceae & Salvia gracilis Benth. & $\mathrm{AR}$ & 1 & EMFS 9 \\
\hline Lamiaceae & Salvia lavanduloides Kunth & $\mathrm{AR}$ & 1 & GIFC 91 \\
\hline Lamiaceae & Salvia sp. L. & HS & 1 & FRPM 596 \\
\hline Lamiaceae & Stachys calcicola Epling & HS & 1 & FRPM 625 \\
\hline Myrtaceae & Ugni montana (Benth.) O. Berg & AR & 2 & FRPM 573 \\
\hline Onagraceae & Lopezia hirsuta Jacq. & HS & 1 & EMFS 2 \\
\hline Onagraceae & Fuchsia splendens Zucc. & $\mathrm{AR}$ & 1 & FRPM 509 \\
\hline Onagraceae & Fuchsia striolata Lundell & AR & 1 & EMFS 11 \\
\hline Orobanchaceae & Castilleja integrifolia var. alpigena L.O. Wiliams & HS & 2 & FRPM 616 \\
\hline Orobanchaceae & Lamourouxia dependens Benth. & HS & 1 & FRPM 619 \\
\hline Orobanchaceae & Lamourouxia xalapensis Kunt & HS & 1 & FRPM 22 \\
\hline Oxalidaceae & Oxalis alpina (Rose) Rose ex R. Kunth & $\mathrm{HI}$ & 3 & FRPM 630 \\
\hline Phrymaceae & Hemichaena fruticosa Benth. & HI & 1 & FRPM 383 \\
\hline Phytolaccaceae & Phytolacca icosandra L. & HS & 2 & FRPM 671 \\
\hline Plantaginaceae & Penstemon gentianoides (Kunth) Poir. & HS & 1 & FRPM 1010 \\
\hline Polygalaceae & Monnina xalapensis Kunt & HS & 1 & FRPM 608 \\
\hline Polygonaceae & Rumex acetosella $\mathrm{L}$. & $\mathrm{AR}$ & 6 & FRPM 603 \\
\hline Ranunculaceae & Ranunculus geoides Kunt ex DC. & HS & 1 & FRPM 598 \\
\hline Rhamnaceae & Ceanothus azureus Desf. ex DC. & AR & 1 & FRPM 580 \\
\hline Rosaceae & Acaena elongata $\mathrm{L}$. & $\mathrm{AR}$ & 2 & FRPM 623 \\
\hline Rosaceae & Alchemilla guatemalensis Rothm. & $\mathrm{HI}$ & 1 & EMFS 14 \\
\hline Rosaceae & Alchemilla pectinata Kunth & $\mathrm{HI}$ & 2 & FRPM 41 \\
\hline Rosaceae & Alchemilla procumbens Rose & $\mathrm{HI}$ & 2 & FRPM 634 \\
\hline Rosaceae & Alchemilla vulcanica Schltdl. \& Cham. & $\mathrm{HI}$ & 2 & FRPM 25 \\
\hline Rosaceae & Holodiscus argenteus (L.f.) Maxim. & AR & 2 & FRPM 564 \\
\hline Rosaceae & Prunus serotina Ehrh. & $\mathrm{AB}$ & 4 & S.N. \\
\hline Rosaceae & Rubus trilobus Moc. \& Sessé ex Ser. & AR & 1 & FRPM 7 \\
\hline Rubiaceae & Houstonia serpyllacea (Schltdl.) C.L. Sm. ex Greenm. & $\mathrm{HI}$ & 1 & FRPM 5 \\
\hline Rubiaceae & Galium mexicanum Kunth & HS & 4 & FRPM 34 \\
\hline Scrophulariaceae & Buddleia skutchii C.V. Morton & $\mathrm{AR}$ & 1 & FRPM 567 \\
\hline Scrophulariaceae & Buddleia megalocephala Donn.Sm. & $\mathrm{AR}$ & 1 & S.N. \\
\hline Solanaceae & Cestrum guatemalense Francey & $\mathrm{AR}$ & 1 & FRPM 32 \\
\hline Solanaceae & Lycianthes gongylodes J.L. Gentry & $\mathrm{AR}$ & 1 & FRPM 574 \\
\hline Solanaceae & $\begin{array}{l}\text { Solanum agrimoniifolium (Ruiz \& Pav. ex. Dunal) J.F. } \\
\text { Macbr. }\end{array}$ & HS & 1 & FRPM 646 \\
\hline Solanaceae & Solanum clarum Correll & $\mathrm{HI}$ & 1 & GIFC 74 \\
\hline Solanaceae & Solanum nigricans $\mathrm{M}$. Martens \& Galeotti & HS & 1 & FRPM 574 \\
\hline
\end{tabular}

Estratos: HI: Herbáceo inferior, HS: Herbáceo superior, AR: Arbustos y AB: Árboles.

Distribución geográfica: 1: Centro de México hasta Centroamérica, 2: México hasta Sudamérica, 3: Estados Unidos de Norteamérica hasta Centroamérica, 4: América, 5: Amplia distribución y 6: Guatemala hasta Sudamérica.

* Muestras de las especies quedaron depositadas en el herbario BIGU de la Escuela de Biología, Facultad de Ciencias Químicas y Farmacia, Universidad de San Carlos de Guatemala. 
